\title{
عزل وتعريف واختبار كفاءة بعض الفطريات في تحلل الهيدروكريون من التربة الملوثة بالنفط
}

\author{
سعاد محمد خليفة أبوالغيث 1 وأحلام القمودي محمد زعيط²

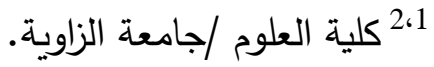 \\ ${ }^{1}$ Alsaadh69alzawia@gmail.com
}

الملخص

استهدفت هذه الدراسة عزل بعض أنواع الفطريات من التربة الملوثة بالهيدروكربون بمصفاة الزاوية Rhizopus, Aspergillus fumigatus, لتكرير النفط، حيث تم عزل وتعريف بعض الفطريات مثل Aspergillus niger, Aspergillus flavus, Aspergillus nidulans أن تواجد وتنوع فطر Aspergillus قد تفوق معنويا مقارنة بتواجد وتنوع فطر Rhizopus. كما تم في هذه الدراسة اختبار قدرة وكفاءة الفطريات المعزولة على النمو واستغلال المركبات الهيدروكربونية المتمثلة في زيت الحمادة وزيت الثرارة بتركيز 1\% و3\%، حيث أوضحت النتائج بأن جنس Rhizopus سجل أعلى معدل للنمو على الوسط الغذائي Malt Extract Agar، وسجل كلا من فطر A. fumigatus وفطر A. nidulans معدّل النمو القطري أعلى معنويا من النمو القطري لفطر A. flavus وفطر هذه المعدّلات العالية تدل على إمكانية استخدام الفطريات المعزولة في المعالجة البيولوجية للتربة الملوّثة بالنفط. الكلمات الرئيسية: Aspergillus - كفاءة التحلّل - فطريات التربة- النمو القطري -الهيدروكربونات. (المقدمة

تتعرض العناصر البيئية إلى اختلال شديد في توازنها نتيجة للاستخدام المستمر للنفط ومشتقاته (بن صادق،1999) ويمكن أن يتسرب النفط بثكل مباشر وغير مباشر إلى داخل البيئة مما يؤدي إلى تلوثها 
الخطير (Thouand et al., 1999) وغالبا ما تؤدي إلى حدوث اضطرابات هائلة للكائنات الحيّة وغير الحيّة (Muller et al., 1992)، وتعدّ التربة ملوّثثة باحتوائها على مادة أو عدة مواد بتركيزات تثكل خطراً على صحة الإنسان والحيوان والنبات، وكما أشارت بعض الدراسات إلى أن النبات يتأثر سلباً عند تلوّث التربة بالنفط الخام، حيث يتسبب التلوث بتأخر وانخفاض نسبة الإنبات بدرجة كبيرة وخاصة في وجود مستوى عالٍ من التلوّث وحدوث نقص تام في تغذية النبات (Schwendinge,1968)، كما اتضح أيضا إلى أن الاستصلاح السريع للتربة الملوثة بالنفط الخام يتم بعملية تتشيط للكائنات الحيّة الدقيقة التي تستعمل الهيدروكربونات الموجودة في النفط كمصدر للكربون والطاقة (أبوالغيث، 2010).

تعدّ الهيدروكربونات النفطية لاسيّما الأروماتية منها Aromatic hydrocarbons من الملوثات البيئية الخطيرة من خلال انتقالها في السلسلة الغذائية (محمد وشعيب،2017) فضلا عن سميتها للأحياء وفعلها المطفر Mutagenic، والمسرطن Carcinogenic وصفاتها الكيميائية والفيزيائية المعقدة التي تجعل منها صعبة التحليل (الطائي وآخرون ،2016)، وتتوفر حالياً عدة طرق لإزالة أو معالجة الملوّثات النفطية بالطرق الكيميائية والفيزيائية والحيوية.

وتحظى المعالجة الإحيائية Bioremediation باهتمام كبير كونها طريقة صديقة للبيئة وعواملها تعرف بأنها إمكانية استخدام الكائنات الحية الدقيقة في هلم وإزالة السمية بطريقة فعالة ولاسيما بالمعالجة الفطرية Mycoremediation التي هي جزء من النظام البيئي وغير مكلفة اقتصادياً، فضلا عن كونها Hameed et تزيل الملوثات دون أن تخلف بقايا منها وتفككها إلى نواتجها النهائية الطبيعة في البيئة (al., 2013) و (Alwan \& Seker, 2010)، وقد زاد الاهتمام بالفطريات لاستخدامها في المعالجة الحيوية للنفط الخام والملوثات البيئية الأخرى حيث تمتلك نظاما أنزيميا معقداً يزيد من قابليتها على تكسير أو تحويل عدد كبير من الملوّثات الخطرة على الإنسان والبيئة (الدوسري وآخرون، 2009)، وتعدّ الفطريات من أهم الأحياء المجهرية الموجودة في التربة والتي تعمل على تكسير العديد من الملوثات ومنها الهيدروكربونات النفطية (حميد، 2015)، وبما أن ليس هناك في ليبيا دراسات حول تنوّع العزلات الفطرية التي قد تكون لها كفاءة في تحلل الملوّثات كان الغرض من الدراسة عزل وتثخيص الفطريات من التربة الملوثة بالنفط واختبار كفاءة الفطريات المعزولة في تحلل الهيدروكربونات معملياً. 


\section{تجميع عيّات التربة بالمنطقة الملوّثة}

أجريت التجربة خريف 2019 في المركز الوطني للصحة الحيوانية بالزاوية لعزل وتعريف أنواع الفطريات النامية على تربة ملوّثة بالهيدروكربون بصفاة الزاوية لتكرير النفط. تم تجميع عيّنات التربة الملوثة بالهيدروكربونات من مصفاة الزاوية لتكرير النفط وبعمق من 5-15 سم، وكان وزن العينة حوالي 1 كجم حيث تم وضعها في أكياس بلاستيكية معقدة وكتب عليها رقم العينة. نقلت العينات إلى المختبر لإجراء الاختبارات الميكروبيولوجية للحصول على عزلات فطرية واختبار كفاءتها في إزالة الهيدروكربونات باستغلالها كمصدر للكربون والطاقة.

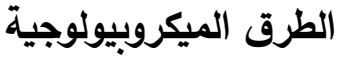

تم تحضير الوسط الغذائي جرام من المسحوق في 1 لتر من الماء المقطر المعقم وعقم في جهاز التعقيم Autoclave بدرجة حرارة 1210م لمدة 15 دقيقة. تم العزل بطريقتين حيث تم أخذ 1 جرام من التربة الجافة الملوثة بالهيدروكربون ووضعها في أنبوبة اختبار تحتوي على 9 مل من الماء المقطر المعقم وتم رجها لمدة 5 دقائق، ثم نقل كمية من الراشح بواسطة إبرة تلقيح معقدة وزرعت على الأطباق المجزة بالوسط الغذائي بطريقة النشر ، حُضنت الأطباق في درجة حرارة 25×2 م بواقع 3 مكررات لكل معاملة.

وفي الطريقة الأخرى للعزل تم نثر 1 جرام من عينة التربة الجافة في طبق بتري معقم بقطر 9 سم حاوي على الوسط الزرعي (MEA) السابق التحضير وبعدها حُضنت الأطباق بدرجة حرارة 25 25 مْ بواقع 3 مكررات للعينة، بعد عملية التحضين للأطباق في كلتا الطريقتين لمدة من 3-5 أيام فحصت النموات الفطرية المختلطة ومنها يتم نقل من كل مستعمرة بواسطة إبرة التلقيح المعقدة على الوسط الغذائي الصلب للحصول على مزارع نقية للتعرف عليها. 
شخصت العزلات الفطرية اعتماداً على المظهر الخارجي للمستعمرة Morphological Feature مثل الثكل واللون وأيضا اعتماداً على الصفات المجرية Microscopic Feature مثل شكل وحجم ولون وتركيب الحوامل والأبواغ والتراكيب الأخرى باستخدام المفاتيح التصنيفية الواردة في المصادر التي تناولت تصنيف ودراسة الفطريات قيد الدراسة الحالية وهي (Domsch et al.,1980) و(Gilman, 1957). استخدام شريط لاصق شفاف بطول 2 سم وذلك بملامسة ووضع الجهة اللاصقة للشريط على سطح المستعمرة الفطرية النامية على الطبق، ثم رفع الشريط على الثريحة الزجاجية الحاوية على قطرة من صبغة القطن الأزرق، ثم تفحص تحت المجهر لمعرفة ترتيب وحجم وشكل الكونيديات.

\section{اختبار كفاءة الفطريات المعزولة على تحلل المركبات الهيدركربونية}

تم اختبار كفاءة الفطريات المعزولة من التربة الملوثة موضوع الدراسة بمدى قدرتها على النمو في أوساط ملوثة بالنفط الخام (زيت الحمادة وزيت الشرارة) وبتركيز 1\% و3\%، ومقارنة أيهما أكثر كفاءة على الى النمو، حيث حُضر الوسط الغذائي (MEA) بإضافة تركيز 1\% و3\% لزيت الحمادة وزيت الثرارة عند درجة حرارة 45م، مع إضافة مضاد بكتيري، ثم مزج الخليط باستخدام المغناطيس المعقم قبل صبه في الأطباق للحصول على تراكيز متجانسة ومتماثلة في كل طبق، ثم لُقحت الأطباق بالخيط الفطري المعزول بواسطة الثاقب الفليني المعقم ذي قطر 5 ملم أُخذ من مستعمرة فطرية نامية لمدة أسبوع، وحضنت الأطباق في درجة حرارة 25 مُ، وتم قياس أقطار المستعرات النامية بعد 7 أيام من التحضين باستخدام measuring tap وقورنت بطبق الكنترول.

استعملت طريقة ANOVA لتحليل البيانات إحصائيا عند مستوى معنوية أقل من (0.05) باستخدام نظام (SPSS) Statistical Packages For Social Sciences.

\section{النتائج والمناقشة}

أوضحت نتائج الفحص والعزل من التربة الملوثة بالهيدروكربون في هذه الدراسة و كما هو موضح بالجدول (1). 
جدول (1): يبين المعزولات الفطرية التي تم عزلها من التربة الملوثة بالنفط

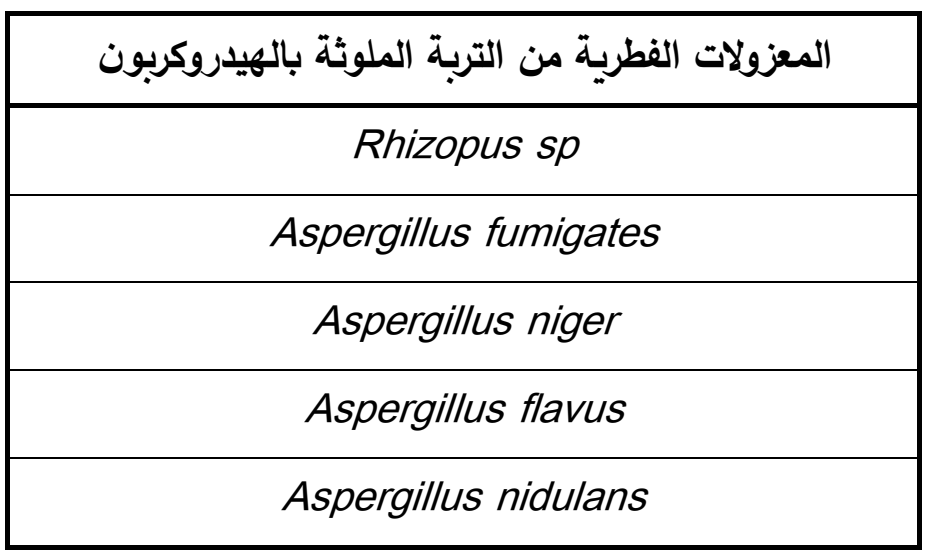

Rhizopus sp., Aspergillus fumigatus Aspergillus niger, تم عزل العديد من الفطريات مثل Aspergillus flavus, Aspergillus nidulans, القطري في جميع التركيزات وكان متوسط النمو 9 سم واتتقت هذه النتائج مع ما أشار إليه ( Dawood Rhizopus بأنه تم عزل العديد من الفطريات من التربة الملوثة بالنفط من بينها فطر (et al.,2015 sp. s spergillus فينما كانت نسبة التتوع وتواجد فذه الدراسة عالية جدا مقارنة بفطر Rhizopus، وهذا ما أكدته الدراسة التي قام بها (Al-Nasrawi,2012) في المكسيك بعزل 16 عزلة فطرية من بينها فطر Aspergillus niger, لما لله من القدرة على النمو في أوساط حاوية على المركبات الهيدروكربونية ويعزى هذا لما يتميز به هذا الفطر من قابلية النمو في بيئات مختلفة وقابليته الأنزيمية العالية التي تمكنه من السيادة على بقية الأجناس الأخرى، وتتفق هذه النتائج مع ما أكده (حميد وآخرون، 2013) بأن . Aspergillus spp أكثر الفطريات سيادة مقارنة بالفطريات التي تم عزلها،

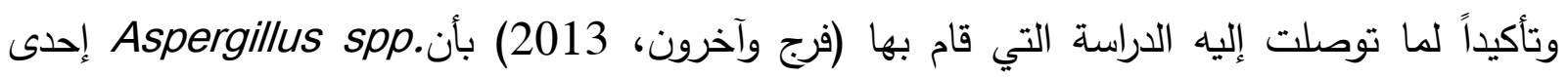
الفطريات التي تم عزلها من التربة الملوثة بالهيدروكربون، كما وتتقق كذلك لما أشار إليه (شفيق ومزعل، 2009) بأن هناك تباين في نسب وانتشار العزلات الفطرية حيث كانت السيادة للفطر Aspergillus . 
جدول (2): تأثير تركيز زيت الحمادة على النمو القطري للفطريات حسب التركيز

\begin{tabular}{|c|c|c|c|}
\hline Sig & المتوسط & تركيز الزيت & نوع الفطر \\
\hline \multirow{3}{*}{0.000} & 6.25 & Control & \multirow{3}{*}{ A. fumigatus } \\
\hline & 6.02 & $\% 1$ & \\
\hline & 5.77 & $\% 3$ & \\
\hline \multirow{3}{*}{0.048} & 4.00 & Control & \multirow{3}{*}{ A. niger } \\
\hline & 0.373 & $\% 1$ & \\
\hline & 4.65 & $\% 3$ & \\
\hline \multirow{3}{*}{0.022} & 7.15 & Control & \multirow{3}{*}{ A. flavus } \\
\hline & 7.18 & $\% 1$ & \\
\hline & 6.88 & $\% 3$ & \\
\hline \multirow{3}{*}{0.002} & 4.65 & Control & \multirow{3}{*}{ A. nidulans } \\
\hline & 4.23 & $\% 1$ & \\
\hline & 4.27 & $\% 3$ & \\
\hline
\end{tabular}

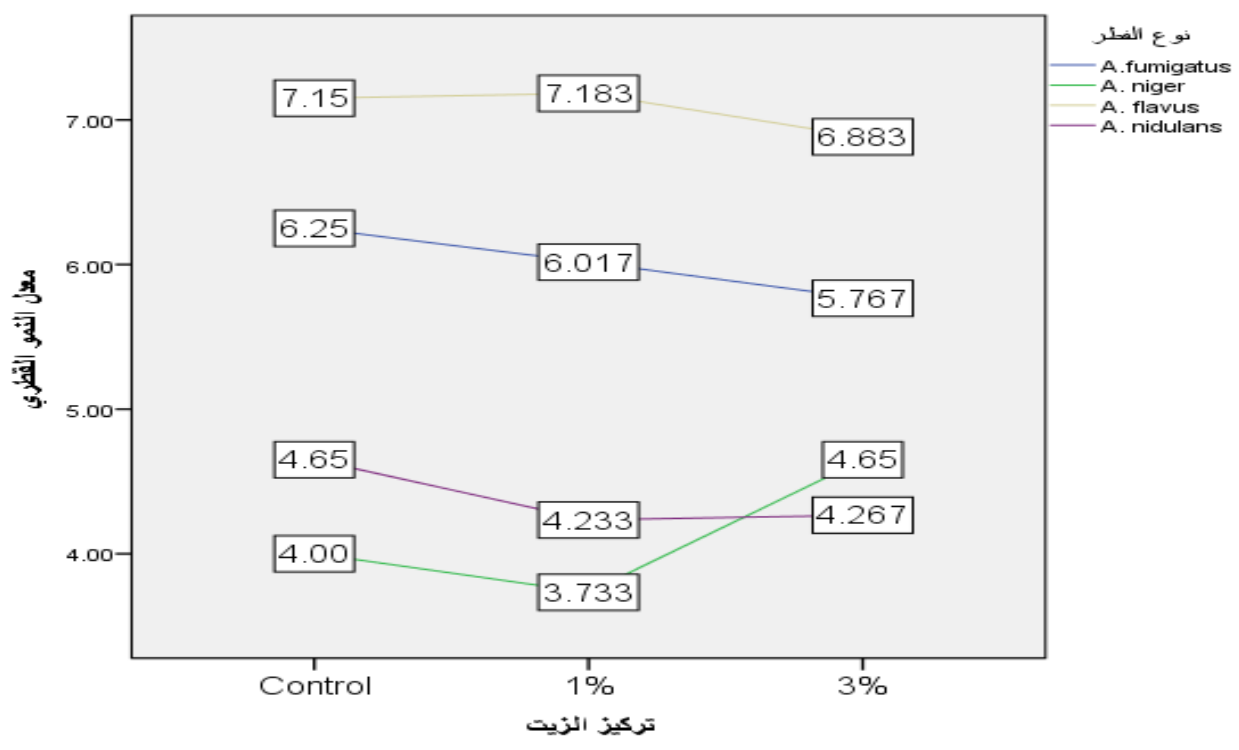

شكل (1): يبين تأثير تركيز زيت الحمادة على النمو القطري للفطريات حسب التركيز. 
جدول (3): تأثير تركيز زيت الثرارة على النمو القطري للفطريات حسب التركيز

\begin{tabular}{|c|c|c|c|}
\hline Sig & المتوسط & تركيز الزيت & نوع الفطر \\
\hline \multirow{3}{*}{0.046} & 6.30 & Control & \multirow{3}{*}{ A. fumigatus } \\
\hline & 5.97 & $\% 1$ & \\
\hline & 5.97 & $\% 3$ & \\
\hline \multirow{3}{*}{0.000} & 2.20 & Control & \multirow{3}{*}{ A. niger } \\
\hline & 5.20 & $\% 1$ & \\
\hline & 4.65 & $\% 3$ & \\
\hline \multirow{3}{*}{0.045} & 7.00 & Control & \multirow{3}{*}{ A. flavus } \\
\hline & 6.87 & $\% 1$ & \\
\hline & 6.75 & $\% 3$ & \\
\hline \multirow{3}{*}{0.001} & 4.75 & Control & \multirow{3}{*}{ A. nidulans } \\
\hline & 5.32 & $\% 1$ & \\
\hline & 4.02 & $\% 3$ & \\
\hline
\end{tabular}

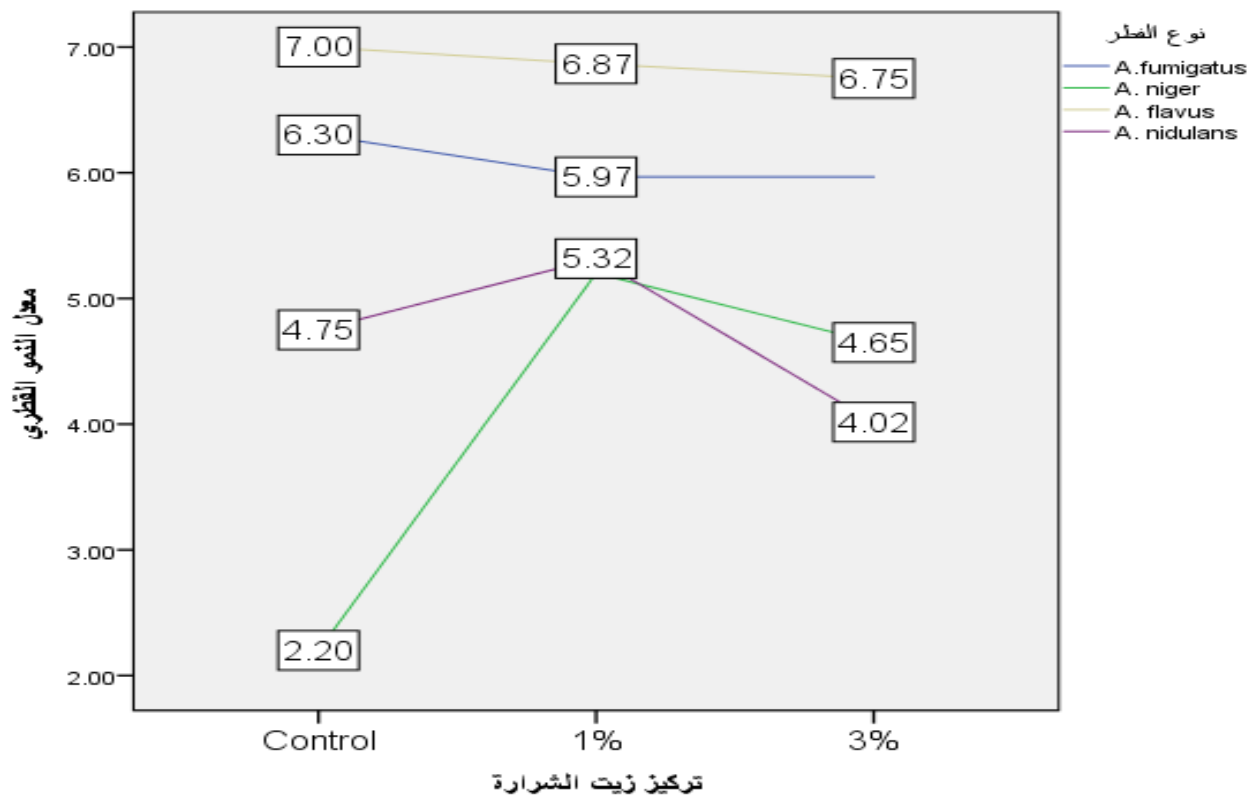

شكل (2): يبين تأثير تركيز زيت الثرارة على النمو القطري للفطريات حسب التركيز. 
استخدمت في هذه الدراسة المركبات الهيدروكربونية متمثلة في زيت الحمادة وزيت الشرارة لمعرفة مدى الاختلاف في التأثير على النمو القطري للفطريات المعزولة، حيث سجلت النتائج بأن فطر Rhizopus sp. الشرارة وفي كل التركيزات المختبرة، وكما هو موضح بالجدول(3،2) والثكل (2،1) أن معدل النمو القطري لفطر A. fumigatus أعلى معنوياً لمجموعة الكنترول (6.25،6.30) من الهموعة ذات التركيز 1\% (6.02،5.97) و\%3 (5.77،5.97) لزيت الشرارة وزيت الحمادة على التوالي، بينما كان معدل النمو القطري أعلى معنويا عند تركيز 1\% من تركيز 3\% (5.77،6.02) لزيت الحمادة، ولا يوجد فرق معنوي بين التركيز 1\% و3\% لمعدل النمو القطري لهذا الفطر لزيت الشرارة (5.97،5.97)،

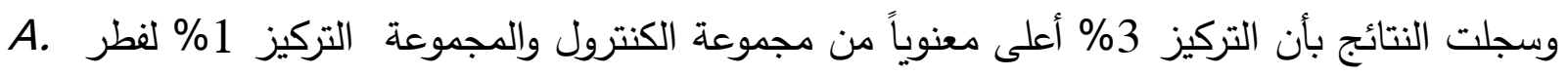
niger لزيت الحمادة (4.65،0.373،4.00) بينما سجل مجموعة التركيز 1\% أعلى معنوياً لمعدل النمو القطري لهذا الفطر من مجموعة الكنترول ومجموعة التركيز 3\% لزيت الشرارة(4.20،205،20.20)، ومعدل النمو القطري لفطر A. flavus في مجموعة الكنترول كانت أعلى معنوياً من مجموعة التركيز3\% (6.88،7.15) ولكن لا توجد فروق معنوية للنمو القطري لهذا الفطر في التركيز 1\% (7.18،7.15) بينما كان معدل النمو القطري في التركيز 1\% أعلى معنويا من تركيز 3\% لزيت الحمادة (6.88،7.18)، بينما عند استخدام زيت الثرارة وجد أن معدل النمو القطري لهذا الفطر لمجموعة الكنترول أعلى معنويا من معدل النمو القطري عند تركيز 3\% (6.75،7.00) ولكن الفروق ليست معنوية عند الكنترول والتركيز 1\% (6.87،7.00) وكذلك لا توجد فروق معنوية بين معدل النمو القطري A. nidulans عند تركيز 1\% و3\% (6.75،6.87)، وأوضحت النتائج أن معدل النمو القطري لفطر لمجموعة الكنترول أعلى معنويا من مجموعة التركيز 1\% و3\% (4.27،4.23،4.65) ولا توجد فروق معنوية بين التركيز 1\% و3\% لزيت الحمادة (4.27،4.23)، وسجل هذا الفطر عند استخدام زيت الشرارة معدل النمو القطري لمجموعة الكنترول أعلى معنويا من مجموعة التركيز 3\% (4.02،45) وكما سجلت مجموعة التركيز 1\% معدل النمو القطري أعلى معنويا من مجموعة الكنترول والتركيز 3\% (4.02،4.75،5.32) بأن Aspergillus) وهذا ما أشار إليه (ALawash, 2019) له القدرة العالية على النمو وإزالة الهيدروكربون من التربة وذلك لقدرته على الاستحلاب والامتزاج في هذه الأوساط. 
جدول (4): يبين تأثير تركيز الزيت على النمو القطري للفطريات حسب نوع الفطر

\begin{tabular}{|c|c|c|c|}
\hline Sig & المتوسط & تركيز الزيت & نوع الفطر \\
\hline \multirow{3}{*}{0.000} & 6.28 & Control & \multirow{3}{*}{ A. fumigatus } \\
\hline & 5.99 & $\% 1$ & \\
\hline & 5.87 & $\% 3$ & \\
\hline \multirow{3}{*}{0.009} & 3.10 & Control & \multirow{3}{*}{ A. niger } \\
\hline & 4.47 & $\% 1$ & \\
\hline & 4.65 & $\% 3$ & \\
\hline \multirow{3}{*}{0.019} & 7.08 & Control & \multirow{3}{*}{ A. flavus } \\
\hline & 7.03 & $\% 1$ & \\
\hline & 6.82 & $\% 3$ & \\
\hline \multirow{3}{*}{0.023} & 4.70 & Control & \multirow{3}{*}{ A. nidulans } \\
\hline & 4.78 & $\% 1$ & \\
\hline & 4.14 & $\% 3$ & \\
\hline
\end{tabular}

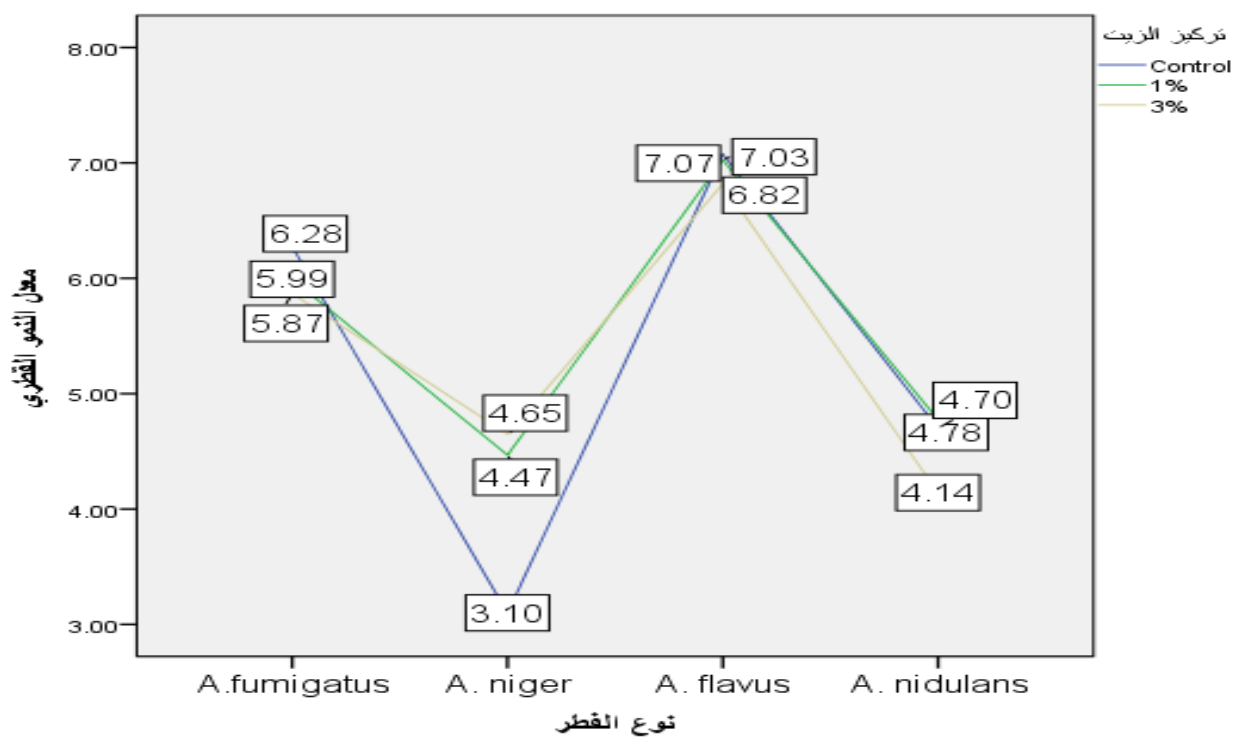

شكل (3): يبين تأثير تركيز الزيت على النمو القطري للفطريات حسب نوع الفطر. 
أظهرت هذه الدراسة نتائج الاختبار لأكثر الفطريات التي تم عزلها نمواً وكفاءة في استغلال الهيدروكربون وتقليل من نسبة تلوثها للتربة فوجد أن فطر .Rhizopus sp سجل أعلى معدل للنمو القطري لهذا A. الفطر 9 سم أي الدرجة القصوى عند كل التركيزات مختبرة الكنترول وعند تركيز 1\% و3\% ويليه فطر كما هو موضح بالجدول (4) والثكل (3)، حيث أن معدل النمو القطري لهذا الفطر أعلى معنوياً

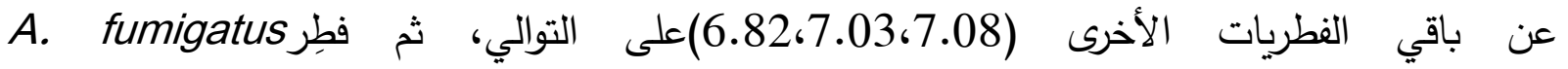
(5.87، nidulans ميث سجل أعلى معدل للنمو القطري من فطر A. niger في الكنترول وتركيز 1\% (4.47،4.78) بينما سجل الأخير أعلى معدل للنمو القطري من الفطر A. nidulans عند تركيز 3\% (4.14،4.65). وما أشارت إليه هذه النتائج يعدّ دليلاً على قدرة الفطريات على النمو في الأوساط الحاوية على الهيدروكربون بنسب متفاوتة وفي هذا تأكيد لما توصلت إليه دراسة سابقة أوضحت بأن نمو الفطريات وبقائها حية حتى بعد 40 يوماً كان واضحاً لاستغلالها للزيت كمصدر للكربون وأن هذا التباين نتيجة لاختلاف الصفات الوراثية لتلك المعزولات (فرج وآخرون، 2013).

\section{الخلاصة والتوصيات}

A. fumigatus, A. niger, A. ) سجلت هذه الدراســـة عزل وتعـريف لبعض الأنواع الفطرية (flavus, Rhizopus, A. nidulans, التربة الملوثة بالنفط، وكثفت أن بعض الأنواع الفطرية مثل Rhizopus, Aspergillus لديها القدرة على النمو واستغلال المركبات الهيدروكربونية الملوثة للتربة. وتؤكد الدراسة على إمكانية أن تستخدم هذه الأنواع في المعالجة البيولوجية للتربة الملوثة بالنفط والحد من تلوث النفط في مختلف النظم البيئية، كما توصي الدراسة بأهمية إجراء مزيد من الدراسات لعزل وتحديد مجموعات أخرى من الكائنات الحية الدقيقة الموجودة في المناطق الملوثة بالمخلفات النفطية للتسريع في عملية المعالجة البيولوجية. 
• أبوالغيث، سعاد محمد. (2010). عزل بكتيريا مؤكسدة للهيدروكربونات من البيئة المحلية وتعريفها-

رسالة ماجستير - جامعة الزاوية.

الدوسري، مصطفى عبدالوهاب؛ الامارة، فارس جاسم؛ عبدالله، سمير خلف. (2009). قابلية بعض الفطريات المعزولة من رسوبيات أهوار جنوب العراق على تكسير النفط الخام مختبرياً. المؤتمر العلمي الثالث لإعادة التأهيل الأهوار 13-23.

• الطائي، ميسون صالح؛ حمد، نداء شهاب؛ البكري، جولان جبار صاحب. (2016). دراسة إمكانية إزالة الهيدروكربونات النفطية وبعض الملوثات في مياه المخلفات النفطية لمصفى النجف. مجلة جامعة بابل/علوم الصرفة التطبيقية 1(24):50- 60.

• بن صادق، علي. (1999). التلوث البيئي. جامعة الملك سعود- الرياض- المملكة السعودية-الطبعة

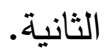

• حميد، مروان سالم. (2015). اختبار قابلية بعض الأنواع الفطرية على النمو في أوساط ملوثة بالنفط الخام -مجلة تكريت للعلوم الصرفة -جامعة تكريت- العراق. 20(5):47-55.

• شفيق، شذى علي؛ مزعل، شيماء نغيش. (2009). تأثير واستغلال النفط الخام من بعض الفطريات المعزولة من التربة. مجلة ابن الهيثم للعلوم الصرفة والتطبيقية- جامعة المستصرية. 22(4).

• فرج، فوزية؛ مؤيد، سرى؛ محمد، رسل. (2013). التحلل الحيوي للمركبات الهيدروكربونية بواسطة الفطريات. مجلة جامعة الكوفة لعلوم الحياة- المؤتمر العلمي الأول للعلوم البيولوجية- جامعة بغداد. 
• محد، فايز عبدالحميد؛ شعيب، فرج محمد. (2017). عزل وتعريف بكتيريا بحرية محللة للنفط الخام

وقياس قدرتها على النمو في تراكيز مختلفة من النفط الخام والمحلية وإمكانية تكوين مستحلب. المجلة الليبية العالمية- جامعة بنغازي - العدد السابع عشر •

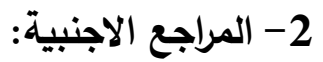

- Al-Hawash AB; Zhang X; Ma F. (2019). Removal and biodegradation of different petroleum hydrocarbons using the filamentous fungus Aspergillus sp.J Microbiologypen. jan 8(1).

- Al-Nasrawi. H. (2012). Biodegradation of Crude Oil by Fungi Isolated from Gulf of Mexico. J Bioremedation \& Biodegradation.3:4.

- Alwan, S. L. and Seker, A. R. (2010). Test the ability of Some Fungi Species in Biodegradation of Some Chemical Pesticides. Kofa University Journal for Life Science. (2) 2.

- Dawood, V.; Madani, M.; Tahmourespour, A. and Golshani, Z. (2015). Hetertropic and Crude Oil Uitilizing Crude Oil Contaminated Regions. J BioremedBiodeg.

- Domsch, K. H.; W. Gams and Anderson,T.H. (1980). Compendium of Soil Fungi Academic Press.859 pp.

- Gilman, J. C. (1957). A manual of soil fungi. Iowa State Univ. Press, Ames, Iowa, USA, (PP.450).

- Hameed, S. M.; Al-khesraji, O. T.; Bander, A.K. (2013). Study of Seasnnal Variation in Fungi Isolated From Petrolium Contaminated Soils In Baiji, Salah adin Province. Tikrit Journal of Pure Science. 18 (5): 114-122.

- Mueller, J.G.; Resnick, S.M.; Shelton,M.E.; Pritchard, P.H.(1992). Effect of inoculation on the biodegradation of weathered Prudhoe Bay crude oil. J.Indstrial Microbiology. 10: 95-102.

- Schwendinger, R. B. (1968). Reclamation of soil contaminated with oil. J. Instrial Petroleum. 54:182-197. 
- Thouand, G.; Bauda, P.; Oudot, J.; Kirsch, G.; Sutton,C .; Vidalie, J.F. (1999). Laboratory evaluation of crude oil biodegradation with commercial or natural microbial inocula. Canadian Journal of Microbiology. 45(2): 106115. 